Article

\title{
Relation between Pro-inflammatory Cytokines and Acetylcholine Levels in Relapsing-Remitting Multiple Sclerosis Patients
}

\author{
Marcella Reale ${ }^{1, *}$, Federica de Angelis ${ }^{2}$, Marta di Nicola ${ }^{1}$, Elisabetta Capello ${ }^{3}$, \\ Maria di Ioia ${ }^{4}$, Giovanna de Luca ${ }^{4}$, Alessandra Lugaresi ${ }^{4}$ and Ada Maria Tata ${ }^{2, *}$
}

1 Department of Experimental and Clinical Sciences, University "G. d'Annunzio" Chieti-Pescara, Via Dei Vestini, 3166100 Chieti, Italy; E-Mail: m.dinicola@unich.it

2 Department of Biology and Biotechnologies Charles Darwin, Sapienza, University of Rome, Research Center of Neurobiology D. Bovet, P.le Aldo Moro, 500185 Roma, Italy; E-Mail: federica.deangelis@uniroma1.it

3 Department of Neuroscience, Ophthalmology and Genetics, University of Genova, 16126 Genova, Italy; E-Mail: ecapello@neurologia.unige.it

4 Department of Neuroscience and Imaging, University "G. d'Annunzio" Chieti-Pescara, 66100 Chieti, Italy; E-Mail: a.lugaresi@unich.it (A.L.)

* Authors to whom correspondence should be addressed; E-Mails: mreale@unich.it (M.R.); adamaria.tata@uniroma1.it (A.M.T.); Tel.: +39-0871-355-4029 (M.R.); +39-06-49912637 (A.M.T.); Fax: +39-0871-355-4029 (M.R.); +39-06-49912351 (A.M.T.).

Received: 1 August 2012; in revised form: 6 September 2012 / Accepted: 27 September 2012 / Published: 3 October 2012

\begin{abstract}
Multiple sclerosis (MS) is a chronic inflammatory, demyelinating and neurodegenerative disorder. Since acetylcholine (ACh) is known to participate in the inflammatory response, we investigated the possible relationship between pro-inflammatory cytokines and acetylcholine levels in relapsing-remitting multiple sclerosis (RR-MS) patients. Levels of ACh and pro-inflammatory cytokines IL1- $\beta$ and IL-17 were measured both in cerebrospinal fluid (CSF) and sera of 22 RR-MS patients in the relapsing phase and in 17 control subjects affected by other non-neurological diseases (OND). We observed higher levels of pro-inflammatory cytokines such as IL-1 $\beta$ and IL-17 in both CSF and serum of RR-MS patients compared to control subjects. Moreover, ACh levels were lower in CSF and serum of RR-MS patients compared to levels of control subjects. Although the relationship between high inflammatory cytokine levels and low
\end{abstract}


ACh levels need to be further investigated in the future, our data suggest that IL-1 $\beta$, and cytokines induced by it, such as IL-17 and ACh, may be involved in the pathogenesis of MS.

Keywords: acetylcholine; pro-inflammatory cytokines; multiple sclerosis

\section{Introduction}

Multiple sclerosis (MS) is characterized by an altered balance of pro- and anti-inflammatory cytokines that causes a high inflammatory state in the central nervous system (CNS), followed by selective destruction of myelin. Inflammatory cytokines as well as oxidative agents are present in MS lesions [1,2], suggesting that the increased production of these toxic molecules may impair cellular defense mechanisms, facilitating demyelination and counteracting remyelination. IL-17 is one of the inflammatory cytokines secreted mainly by activated T cells and similarly to TNF- $\alpha$ and IL-1, IL-17 has pro-inflammatory properties, playing a key role in the pathogenic mechanisms of MS [3].

Thus, the control of inflammation and neuroprotection should be the key points in designing MS therapeutic protocols. Novel research tools and therapeutic approaches are oriented towards the identification of new molecules that may decrease the level of the noxious agents, and at the same time stimulate oligodendrocyte (OL) progenitors to proliferate and differentiate.

Among the endogenous molecules that may have neuroprotective effects there are the neurotransmitters and, in particular, acetylcholine (ACh). In fact, it has been demonstrated that ACh reduces the inflammatory state as indicated by chronic administration of selective acetylcholinesterase inhibitors (AChEI) in autoimmune encephalomyelitis (EAE) animal models [4]. Several ACh receptor subtypes (muscarinic and nicotinic types) are expressed by OLs and their precursors. As recently demonstrated, the muscarinic agonists are able to induce oligodendrocyte progenitor cell (OPC) proliferation and inhibit the expression of myelin proteins (e.g., myelin basic protein) in mature OLs [5]. On the other hand, the selective stimulation of M2 receptors causes a decrease of OPC progenitor survival, suggesting that the levels of $\mathrm{ACh}$ present in the environment and the set of muscarinic receptor subtypes expressed by each subject might play a relevant role in the control of OL progenitor survival, proliferation and differentiation [5].

The involvement of ACh in the modulation of inflammatory states has also been reported. T-cells express both muscarinic (mAChRs) and nicotinic (nAChRs) receptors. Their activation produces differential effects since muscarinic receptors enhance pro-inflammatory mediators, while nicotinic receptor activation exerts an anti-inflammatory response [4,6]. In fact, muscarinic receptor stimulation can elicit upregulation of c-fos, nitric oxide synthase and IL-2 production [6]. On the other hand, ACh, through the $\alpha 7$ nicotinic receptors, can inhibit the release of TNF $\alpha$ and IL-1 from macrophages, suggesting the existence of a "cholinergic anti-inflammatory pathway" [7]. Moreover, it has also been demonstrated that human $\mathrm{T}$ cells can synthesize $\mathrm{ACh}$, suggesting that it may be an autocrine signal modulating $\mathrm{T}$ cell-dependent immune response [8,9]. Although it is not known whether ACh participates in the immune system activity, it is now evident that lymphocytes and macrophages express an independent cholinergic system $[4,8]$. 
In consideration of these data, we investigated the possible involvement of $\mathrm{ACh}$ in the inflammatory state of MS patients, with the hypothesis that ACh levels are reduced in active MS thereby leading to heightened inflammation. For this purpose we measured the cytokine production and ACh levels in CSF and sera of Relapsing-Remitting (RR)-MS patients and in control subjects affected by other neurological diseases.

\section{Results and Discussion}

\subsection{Cytokine Levels}

In the group of 22 RR-MS subjects in the relapsing phase, the analysis of cytokines showed that IL-17 levels in serum were significantly elevated compared to control subjects $(p<0.05)$, while in the CSF the levels were lower than in the control group although the differences were not statistically significant $(p=0.201)$. However, in both RR-MS and control group, the mean level of IL-17 was higher in the CSF than in serum (Table 1). Similarly, the level of IL-1 $\beta$ in the CSF and serum from RR-MS patients was significantly higher than in control subjects $(p<0.05)$. In the group of RR-MS subjects, levels of serum IL- $1 \beta$ were about four times higher than CSF levels $(p=0.021)$ (Table 1$)$.

We also measured the levels of IL-10, TNF- $\alpha$ and IL-4 both in CSF and sera and we did not find statistical differences between RR-MS patients and control subjects (data not shown).

\subsection{Measurement of Acetylcholine Levels}

Since ACh takes part in the modulation of inflammation, we also measured the levels of ACh in the CSF and serum of RR-MS patients. ACh levels were significantly lower in RR-MS patients than in control subjects, both in sera and CSF (Table. 1). In fact, the ACh levels in CSF of RR-MS patients were $124.3 \pm 56.4 \mathrm{pmol} \mathrm{ACh} / \mathrm{mL}$ compared to $393.6 \pm 142.5 \mathrm{pmol} \mathrm{ACh} / \mathrm{mL}$ measured in control subjects $(p<0.05)$. Similarly, in the serum of RR-MS patients, ACh levels were $175.4 \pm 68.9 \mathrm{pmol} \mathrm{ACh} / \mathrm{mL}$ while in the control subjects they were $586.9 \pm 149.4 \mathrm{pmol} \mathrm{ACh} / \mathrm{mL}(p<0.05)$ (Table 1).

No relationship was found between ACh and gender $(p=0.835)$. Regression analysis did not show any statistically significant relationship between ACh levels and main demographic and clinical characteristics of patients, such as age $(p=0.211)$, EDSS $(p=0.471)$ and disease duration $(p=0.582)$.

Table 1. Cytokine and acetylcholine (ACh) levels in serum and cerebrospinal fluid (CSF) (mean \pm standard error).

\begin{tabular}{|c|c|c|c|c|c|c|}
\hline \multirow{2}{*}{ Variable } & \multicolumn{2}{|c|}{ Control group $(n=17)$} & \multirow{2}{*}{$\begin{array}{c}\text { Wilcoxon } \\
\text { U test } \\
p \text {-value }\end{array}$} & \multicolumn{2}{|c|}{ RR-MS group $(n=22)$} & \multirow{2}{*}{$\begin{array}{c}\text { Wilcoxon } \\
\text { U test } \\
p \text {-value }\end{array}$} \\
\hline & Serum & CSF & & Serum & CSF & \\
\hline IL-17 (pg/mL) & $8.3 \pm 2.7$ & $25.7 \pm 2.9$ & 0.144 & $15.7 \pm 2.4^{*}$ & $20.6 \pm 2.9$ & 0.208 \\
\hline IL-1 $\beta(\mathrm{pg} / \mathrm{mL})$ & $19.4 \pm 4.3$ & $6.2 \pm 0.8$ & 0.013 & $97.4 \pm 26.1^{*}$ & $25.0 \pm 7.2^{*}$ & 0.021 \\
\hline $\mathrm{ACh}(\mathrm{pmol} / \mathrm{mL})$ & $586.9 \pm 149.4$ & $393.6 \pm 142.5$ & 0.917 & $175.4 \pm 68.9^{*}$ & $124.3 \pm 56.4^{*}$ & 0.686 \\
\hline
\end{tabular}

Wilcoxon $\mathrm{U}$ test $p$-value relative to comparison serum vs. CSF values within each group; * $p<0.05$;

Mann-Whitney U test relative to comparison RR-MS group vs. Control group 


\section{Discussion}

MS is considered an inflammatory autoimmune disease of the CNS with a variable clinical course. Patients can present with a rapid progression of the disease or remain clinically stable for several decades. The qualitative and quantitative levels of cytokines reflect the inflammatory state of the patient and may affect the disease outcome [10]. Peripheral blood and endothelial cells are well known sources of cytokines, but also brain cytokine over-production, deriving from the CNS, might in fact contribute to the peripheral cytokine pool. On the other hand, peripheral cytokines might affect human brain functions by crossing the blood-brain barrier (BBB) and interacting with the CNS [11]. It is known that an acute inflammatory stimulus (such as IL-1 or endotoxin) in the brain, efficiently induces inflammatory cytokine production in the periphery $[12,13]$. Thus, it seems that the CNS not only is able to produce cytokines, but it may also contribute to increase the peripheral pool. Since cytokines are involved in the regulation of inflammatory responses, their levels may reflect the disease process.

Several studies have analyzed peripheral inflammatory parameters, including cytokines and related molecules in the blood (serum, plasma) of MS patients [14]. The same parameters have been measured less frequently in the CSF. For these reasons we have evaluated the levels of different pro- and anti-inflammatory cytokines both in serum and in CSF of RR-MS patients. Our results confirm the presence, in RR-MS patients, of an altered profile of cytokines typically associated with a strong inflammatory response with high levels of IL-17 in serum and high levels of IL-1 $\beta$ both in serum and in CSF. Comparison of cytokine levels showed higher serum concentrations of the cytokines IL-1 $\beta$ and higher levels of IL-17 in CSF of RR-MS patients. Further studies are needed to establish whether higher levels in the CSF compared to serum of IL-17 could be due to migration of Th17 cells into the CNS, thereby inducing inflammation.

Our data may point out the peripheral/central and inflammatory/regulatory balance which may explain how the timely control of inflammation in the CNS may be a way of limiting peripheral inflammation.

Moreover, several studies have documented the accumulation of Th17 cells in MS lesions, suggesting that Th17 cells may play a central role in the immunopathogenesis of MS $[15,16]$.

It is known that ACh modulates the production and release of pro- and anti-inflammatory cytokines [3]. Recently, it has also been demonstrated that in vivo treatment with AChE inhibitors (e.g., donepezil) down-regulates the expression and production of IL-1 $\beta$, IL-6 and TNF $\alpha$ and up-regulates the expression and production of IL-4 in peripheral blood mononuclear cells (PBMC) of Alzheimer's patients $[17,18]$. Moreover, the AChE inhibitors cause a consistent reduction of CNS lymphocyte infiltrates in autoimmune encephalomyelitis (EAE) animal models ameliorating clinical symptoms [3]. On the other hand, the nicotinic stimulation appears involved in suppression of Th1 and Th17 response [19] and EAE mice, $\mathrm{KO}$ for nicotinic receptor $\alpha 7$, show a significant reduction of the number of $\mathrm{T}$ lymphocytes migrating to the CNS [20]. These data suggest that ACh controls, probably through different acetylcholine receptor subtypes, the cytokine network possibly by acting on the lymphocyte activity and migration [4,6,20]. Given the effects of ACh on cytokine levels, we have measured the levels of ACh in serum and CSF of RR-MS patients. The fluorimetric assay used in the present work, although less sensitive than HPLC method [21], has already been used to measure ACh levels in various biological fluids without pretreatment or purification of samples [22]. This technique has 
shown significant differences of concentrations in CSF and serum among RR-MS patients and control subjects. In fact the data obtained indicate the presence of lower levels of ACh both in serum and in CSF of RR-MS patients compared to subjects affected by other neurological diseases. The decreased ACh level may be, at least in the central nervous system, in part dependent on neuron loss that occurs in MS as a consequence of the myelin and axon degeneration. On the other hand, although we did not find any statistically significant correlation between the low levels of ACh and high levels of cytokines both in serum and CSF (see Figure 1), the decreased levels of ACh in serum of RR-MS patients suggest that ACh levels may be correlated with the high inflammatory state.

Our study, for the first time, investigated ACh levels in biological fluids of RR-MS patients. Interestingly the levels of ACh we found in CSF and serum of RR-MS patients were significantly lower than levels detected in OND (control) patients and significantly different compared to the levels reported in other studies, e.g. in patients treated with analgesic drugs after acoustic neuroma resection [21] or affected by other neurological diseases (e.g., Parkinson's disease, Alzheimer's disease and amyotrophic lateral sclerosis) [23]. We hypothesize that low levels of ACh may correlate with diseases classically characterized by an impairment of ACh neurotransmission, or the treatment with analgesic drugs may directly or indirectly affect the levels of ACh that usually participate in the modulation of nociception stimuli [24]. Finally the different methods used to measure ACh levels [21,22-24] might hamper the comparison between studies on subjects affected by different neurological diseases.

Figure 1. Correlation coefficient between ACh and cytokine levels in serum and CFS of relapsing-remitting multiple sclerosis (RR-MS) patients.

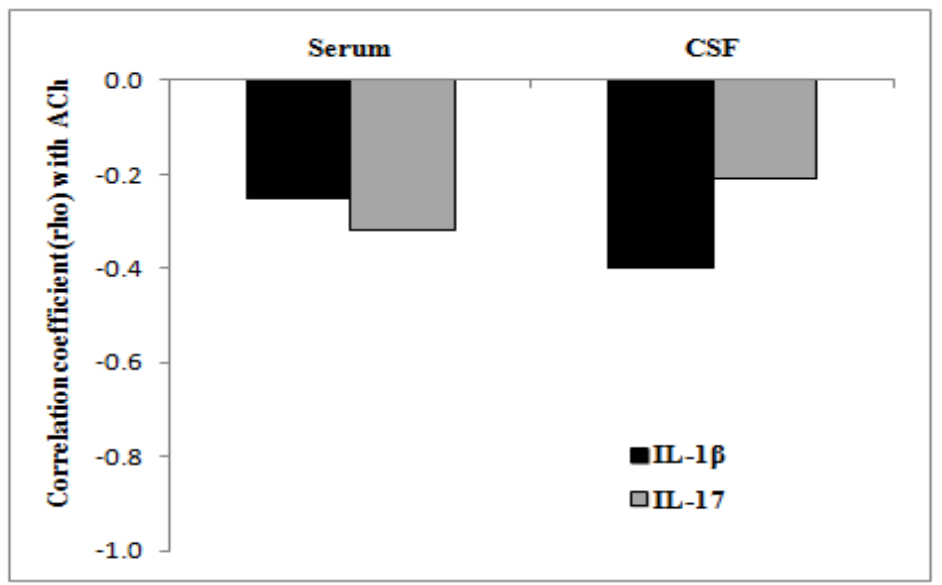

\section{Experimental Section}

\subsection{Patients}

Patients with MS diagnosed according to the revised McDonald criteria [25] and followed by the MS Center of the University "G. d'Annunzio" of Chieti and by the Department of Neuroscience, University of Genova (Italy) were included in the study. The diagnosis of RR-MS was confirmed by brain magnetic resonance imaging (MRI) with gadolinium, and twenty-two RR-MS patients with active disease were enrolled in the present study. In this study, all patients were judged to have active disease according to clinical criteria, that means the appearance of new symptoms or a significant 
aggravation of pre-existing symptoms within the last 1 months. Subjects with other neurological diseases (OND, $n=17$; patients with chronic back pain $(n=6)$, chronic tension headache $(n=5)$, ischemic transverse myelopathy $(n=6)$; were also included in the study as controls. All subjects gave their informed consent in following with the declaration of Helsinki. None of the patients had received corticosteroids or immunosuppressive drugs in the 6 months prior to blood withdrawal. None of the subjects had any inflammatory diseases, in the previous month, that may have been associated with increases in cytokine secretion.

Phlebotomy and lumbar puncture were performed in the same morning between 08:00 and 10:00. Serum obtained from all subjects was centrifuged and aliquots were stored at $-80{ }^{\circ} \mathrm{C}$ until cytokine assays were performed. Lumbar puncture was performed for diagnostic purposes in all cases, and the CSF collected was centrifuged at $2000 \mathrm{~g}$ for $10 \mathrm{~min}$ at $4{ }^{\circ} \mathrm{C}$, aliquoted and were snap-frozen within 20 min after harvesting and stored at $-80^{\circ} \mathrm{C}$ until analysis. A consecutive code number was assigned to each sample to ensure that all assays were performed in a blinded manner. All samples were analyzed not more than sixth months after collection. The main characteristics of the patients involved in the study are summarized in Table 2.

Table 2. Characteristics of the patients involved in this study.

\begin{tabular}{lccc}
\hline Variable & $\begin{array}{c}\text { Control group } \\
(\boldsymbol{n}=\mathbf{1 7})\end{array}$ & $\begin{array}{c}\text { RR-MS group } \\
(\boldsymbol{n}=\mathbf{2 2})\end{array}$ & $\boldsymbol{p}$-value \\
\hline Gender, $n(\%)$ & $2(11.8)$ & $7(31.8)$ & $0.275^{\mathrm{a}}$ \\
$\quad$ Male & $15(88.2)$ & $15(68.2)$ & \\
$\quad$ Female & $41(19-76)$ & $37(18-59)$ & $0.377^{\mathrm{b}}$ \\
Age (years), median (range) & $5.1(2.4-17.3)$ & $5.5(2.8-15.2)$ & $0.986^{\mathrm{b}}$ \\
BBB impairment, median (range) & $4(3-6)$ & $5(3-6)$ & $0.975^{\mathrm{b}}$ \\
Duration of disease (years), median (range) & - & $2.5(0.0-6.0)$ & \\
EDSS, median (range) & - & \\
\hline BBB: Blood-Brain Barrier; EDSS: Expanded Disability Status Scale; ${ }^{\mathrm{a}}$ Fisher's exact test; ${ }^{\mathrm{b}}$ Mann-Whitney U test.
\end{tabular}

\subsection{Cytokine and Acetylcholine Level Measurement}

All cytokines were measured using commercial ELISA (Endogen, USA) kits, in accordance with manufacturer's instructions. All steps were performed in duplicate and at room temperature. Cytokine levels were then calculated plotting the optical density (O.D.) of each sample against the standard curve. Duplicate values that differed from the mean by greater than $10 \%$ were not considered for further analysis.

ACh was measured by commercial colorimetric/fluorimetric kit (Abcam, Cambridge, UK) [22]. Fifty microliter of the sample (CSF or serum) was mixed with $50 \mu \mathrm{L}$ of reaction solution including choline assay buffer, choline probe, enzyme mix and AChE according to the instructions. The level of $\mathrm{Ch} / \mathrm{ACh}$ (pmol/well) was calculated by plotting the fluorescence of each sample in relation to choline standard curve. The standard curve, according to the fluorimetric procedure as indicated by manufacturer's instructions, was obtained by diluting the Choline Standard to generate 0, 10, 20, 30, 40, 50, 100 and $200 \mathrm{pmol} / \mathrm{well}$ of the Choline Standard. The measurement of the fluorescence was 
obtained by Glomax Multi Detection System (Promega) at $\lambda$ Ex/Em 535/587 nm. Considering the intensity of the fluorescence of the samples, the $x$ values (corresponding to $\mathrm{Ch} / \mathrm{ACh}$ concentration and reported as pmol/well) were calculated by the standard curve equation using GraphPad Prism 5.0. The limit of detection of Choline is defined by the analyte concentration resulting in fluorescence higher than that of the dilution medium ( 0 dose of standard choline $=$ Blank $)$.

\subsection{Statistical Methods}

The qualitative variables were summarized as frequency and percentage and quantitative variables as median and range or mean and standard error. The results were reported separately for RR-MS and control groups. Differences in characteristics of the patients and cytokine/ACh levels among RR-MS and control groups were tested by Mann-Whitney U test and Fisher's exact test for continuous and categorical variables, respectively. Differences between serum and CSF values were tested by Wilcoxon U test.

To evaluate the relationship between $\mathrm{ACh}$ and main demographic and clinical characteristics of patients we performed univariate regression analysis or Mann-Whitney $U$ test for quantitative and qualitative variables, respectively. The Spearman rho correlation coefficient was applied to evaluate the correlation between ACh and cytokine levels. Statistical analysis was performed using SPSS ${ }^{\circledR}$ Advanced Statistical 11.0 software (SPSS Inc, Chicago, Illinois, USA).

\section{Conclusions}

The cholinergic anti-inflammatory pathway is a physiological neuro-immune mechanism that regulates innate immune function and controls inflammation inhibiting the production of pro-inflammatory cytokines and chemokines and suppressing the activation of nuclear factor-kappa B expression and oxidative system [26,27]. Although in our RR-MS patients the no significant correlation between cytokine and ACh levels was found (Figure 1), probably due to the relatively low sample number, it is intriguing that $\mathrm{ACh}$ and pro-inflammatory cytokine levels varied in an inverse manner. Considering the ability of ACh to modulate inflammatory responses and OPC proliferation, the decreased levels of ACh observed in RR-MS patients may contribute to maintenance of an inflammatory state and exacerbate the symptoms of MS. Although further analysis will be necessary to elucidate the role of the cholinergic pathway in the pathogenesis of MS, our observations together with the evidence that AChE inhibitors ameliorate the clinical symptoms in EAE models [4] point out a new possible role for ACh in MS.

\section{Acknowledgments}

The work was supported by Italian Multiple Sclerosis Foundation (FISM/AISM) project number 2009/R/29. De Angelis's fellowship was in part supported by FISM and by University of Rome La Sapienza funds. The authors are grateful to G. Augusti Tocco and Nigel H. Greig for scientific discussions and R. Barbacane for the English revision. 


\section{References}

1. Filion, L.G.; Graziani-Bowering, G.; Matusevicius, D.; Freedman; M.S. Monocyte-derived cytokines in multiple sclerosis. Clin. Exp. Immunol. 2003, 131, 324-334.

2. Lock, C.; Hermans, G.; Pedotti, R.; Brendolan, A.; Schadt, E.; Garren, H.; Langer-Gould; A.; Strober, S.; Cannella, B.; Allard, J.; et al. Gene-microarray analysis of multiple sclerosis lesions yields new targets validated in autoimmune encephalomyelitis. Nat. Med. 2002, 8, 500-508.

3. Zepp, J.; Wu, L.; Li, X. IL-17 receptor signaling and T helper 17-mediated autoimmune demyelinating disease. Trends Immunol. 2011, 32, 232-239.

4. Nizri, E.; Hamra-Amitay, Y.; Sicsic, C.; Lavon, I.; Brenner T. Anti-inflammatory properties of cholinergic up-regulation: A new role for acetylcholinesterase inhibitors. Neuropharmacology 2006, 50, 540-547.

5. De Angelis, F.; Bernardo, A.; Magnaghi, V.; Minghetti, L.; Tata, A.M. Muscarinic receptors subtypes as potential target to modulate oligodendrocyte progenitor survival, proliferation and differentiation. Dev. Neurobiol. 2012, 72, 713-728.

6. Kawashima, K.; Fujii, T. The lymphocytic cholinergic system and its contribution to the regulation of immune activity. Life Sci. 2003, 74, 675-696.

7. Rosas-Ballina, M.; Olafsson, P.S.; Ochani, M.; Valdes-Ferrer, S.L.; Levine, Y.A.; Reardon, C.; Tusche, M.W.; Pavlov, V.A.; Andersson, U.; Chavan, S.; et al. Acetylcholine-synthesizing T cells relay neural signals in a vagus nerve circuit. Science 2011, 334, 98-101.

8. Kawashima, K.; Fujii, T. Extraneuronal cholinergic system in lymphocytes. Pharmacol. Ther. 2000, 86, 29-48.

9. Shi, F.D.; Piao, W.H.; Kuo, Y.P.; Campagnolo, D.I.; Vollmer, T.; Lukas, R.J. Nicotinic attenuation of central nervous system inflammation and autoimmunity. J. Immunol. 2009, 182, 1730-1739.

10. Simpson, J.E.; Woodroofe, M.N. Inflammation in the central nervous system in multiple sclerosis: The role of chemokines and their receptors. Inflammopharmacology 2001, 9, 23-33.

11. Banks, W.A.; Robinson, S.M.; Verma, S.; Morley, J.E. Efflux of human and mouse amyloid beta proteins 1-40 and 1-42 from brain: Impairment in a mouse model of Alzheimer's disease. Neuroscience 2003, 121, 487-492.

12 De Simoni, M.G.; Sironi, M.; de Luigi, A.; Manfridi, A.; Mantovani, A.; Ghezzi, P. Intracerebroventricular injection of interleukin 1 induces high circulating levels of interleukin 6. J. Exp. Med. 1990, 171, 1773-1778.

13. DellaGioia, N.; Hannestad, J. A critical review of human endotoxin administration as an experimental paradigm of depression. Neurosci. Biobehav. Reviews 2010, 34, 130-143.

14. Lutterotti, A.; Berger, T.; Reindl, M. Biological markers for multiple sclerosis. Curr. Med. Chem. 2007, 14, 1956-1965.

15. Tzartos, J.S.; Friese, M.A.; Craner, M.J.; Palace, J.; Newcombe, J.; Esiri, M.M.; Fugger, L. Interleukin-17 production in central nervous system-infiltrating $\mathrm{T}$ cells and glial cells is associated with active disease in multiple sclerosis. Am. J. Pathol. 2008, 172, 146-155. 
16. Wang, H.H.; Dai, Y.Q.; Qiu, W.; Lu, Z.Q.; Peng, F.H.; Wang, Y.G.; Bao, J.; Li, Y.; Hu, X.Q. Interleukin-17-secreting $\mathrm{T}$ cells in neuromyelitis optica and multiple sclerosis during relapse. J. Clin. Neurosci. 2011, 18, 1313-1317.

17. Lugaresi, A.; di Iorio, A.; Iarlori, C.; Reale, M.; de Luca, G.; Sparvieri, E.; Michetti, A.; Conti, P.; Gambi, D.; Abate, G.; et al. IL-4 in vitro production is up-regulated in Alzheimer's disease patients treated with acetylcholinesterase inhibitors. Exp. Gerontol. 2004, 39, 653-657.

18. Reale, M.; Iarlori, C.; Gambi, F.; Feliciani, C.; Salone, A.; Toma, L.; de Luca, G.; Salvatore, M.; Conti, P.; Gambi, D. Treatment with an acetylcholinesterase inhibitor in Alzheimer patients modulates the expression and production of the pro-inflammatory and anti-inflammatory cytokines. J. Neuroimmunol. 2004, 148, 162-171.

19. Nizri, E.; Irony-Tur-Sinai, M.; Lory, O.; Orr-Urtreger, A.; Lavi, E.; Brenner, T. Activation of the cholinergic anti-inflammatory system by nicotine attenuates neuroinflammation via suppression of Th1 and Th17 responses. J. Immunol. 2010, 183, 6681-6688.

20. Nicolussi, E.M.; Huck, S.; Lassmann, H.; Bradl, M. The cholinergic anti-inflammatory system limits $\mathrm{T}$ cell infiltration into the neurodegenerative CNS, but cannot counteract complex CNS inflammation. Neurobiol. Diseases 2009, 35, 24-31.

21. De Kock, M.; Eisenacht, J.; Tong, C.; Schmitz, A.L.; Scholts, J.L. Analgesic doses intrathecal but not intravenous clonidine increase Acetylcholine in cerebrospinal fluid in humans. Anest. Analg. 1997, 84, 800-803.

22. Milara, J.; Serrano, A.; Peirò, T.; Amadeu, G.; Miralpeix, M.; Morcillo, E.J.; Cortijo, J. Aclidinium inhibits human lung fibroblast to myofibroblast transition. Thorax 2012, 97, 229-237.

23. Yamada, H.; Otsuka, M.; Fujimoto, M.; Kawashima, K.; Yoshida, M. Determination of acetylcholine concentration in cerebrospinal fluid of patients with neurologic diseases. Acta Neurol. Scand. 1996, 93, 76-78.

24. Dussor, G.O.; Helesic, G.; Hargreaves, K.M.; Flores, C.M. Cholinegic modulation of nociceptive responses in vivo and neuropeptide release in vitro at the level of the primary sensory neuron. Pain 2004, 107, 22-32.

25. Polman, C.H.; Reingold, S.C.; Edan, G.; Filippi, M.; Hartung, H.P.; Kappos, L.; Lublin, F.D.; Metz, L.M.; McFarland, H.F.; O'Connor, P.W.; et al. Diagnostic criteria for multiple sclerosis: 2005 revisions to the "McDonald Criteria". Ann. Neurol. 2005, 58, 840-846.

26. Gallowitsch-Puerta, M.; Pavlov, V.A. Neuro-immune interactions via the cholinergic anti-inflammatory pathway. Life Sci. 2007, 80, 2325-2329.

27. Grando, S.A.; Kawashima, K.; Wessler, I. Introduction: The non-neuronal cholinergic system in humans. Life Sci. 2003, 72, 2009-2012.

(C) 2012 by the authors; licensee MDPI, Basel, Switzerland. This article is an open access article distributed under the terms and conditions of the Creative Commons Attribution license (http://creativecommons.org/licenses/by/3.0/). 